\title{
The BoND-tree: An Efficient Indexing Method for Box Queries in Non-ordered Discrete Data Spaces
}

\author{
Changqing Chen \\ Alok Watve \\ Sakti Pramanik \\ Qiang Zhu
}

\begin{abstract}
Box queries (or window queries) are a type of query which specifies a set of allowed values in each dimension. Indexing feature vectors in the multi-dimensional Non-ordered Discrete Data Spaces (NDDS) for efficient box queries is becoming increasingly important in many application domains such as genome sequence databases. Most of the existing work in this field targets the similarity queries (range queries and k-NN queries). Box queries, however, are fundamentally different from similarity queries. Hence the same indexing schemes designed for similarity queries may not be efficient for box queries. In this paper, we present a new indexing structure specifically designed for box queries in the NDDS. Unique characteristics of the NDDS are exploited to develop new node splitting heuristics. For the BoND-tree, we also provide theoretical analysis to show the optimality of the proposed heuristics. Extensive experiments with synthetic data demonstrate that the proposed scheme is significantly more efficient than the existing ones when applied to support box queries in NDDSs. We also show effectiveness of the proposed scheme in a real world application of primer design for genome sequence databases.
\end{abstract}

Index Terms—Box Query, Non-ordered Discrete Data, Categorical Data, Indexing

\section{INTRODUCTION}

B OX query in NDDS is an important type of query which is defined by specifying a set of allowed values in each dimension. These queries are useful in many diverse applications such as bioinformatics, biometrics, data mining and E-commerce. In general, indexes are used to achieve improved response time for query execution in large databases. In this paper we propose an effective indexing scheme for implementing box queries in NDDS for large databases. There are many existing indexing schemes for large databases for continuous data spaces (CDS). These indexing schemes are not suitable for queries in NDDS because of the fundamental differences between the two spaces. Indexing techniques in the CDS rely on the fact that the indexed values can be ordered in each dimension which is not the case in NDDS. However, NDDS has certain value discrimination properties which can be exploited for efficient implementation of indexes in NDDS. The proposed work exploits these properties of NDDS to develop a new indexing scheme, BoND-tree, targeted towards improving the performance of box queries.

In this paper we focus on the application of box queries for primer design in genome sequence databases. A box query in a genome sequence database of q-grams (fixed length overlapping short sequences created from the database of variable length long genome sequences) allows a set of characters in each position of a q-gram. For example, a box

- C. Chen, A. Watve and S. Pramanik are with the Department of Computer Science and Engineering, 3115 Engineering Building, Michigan State University, MI 48824-1226, USA

Email : \{chencha3,watvealo,pramanik\}@cse.msu.edu

- Q. Zhu is with the Department of Computer and Information Science, University of Michigan, Dearborn, MI 48128, USA

Email : qzhu@umich.edu query in a database of three character long q-grams can be $\{\{A\},\{G, T\},\{C, T\}\}$. This query fetches those q-grams from the database which have the character $\mathrm{A}$ in position one, $\mathrm{G}$ or $\mathrm{T}$ in position two and $\mathrm{C}$ or $\mathrm{T}$ in position three. Thus, the box query is equivalent to searching for four individual search keys $\{A G C, A T C, A G T, A T T\}$.

A primer in molecular biology is a fixed length short sequence (strand of nucleotides) that acts as a terminus for a sub-sequence of a genome sequence. A primer is used to search a database of variable length genome sequences. For search purpose, we can consider genome sequences as a database of q-grams. Developing a good primer is critical in many genome applications. Although a genome sequence contains one of the four characters $\{A, G, T, C\}$ in each position, a primer may allow more than one characters in some positions. Such primers are called degenerate primers.

In the process of primer design, a biologist first generates a set of candidate primers which may be degenerate and then eliminate those which cannot be used, by matching the primer against a database of genome sequences. Traditionally, this search is performed by linearly scanning the genome sequence files. However, an index scheme like the BoNDtree can significantly improve the search performance. A candidate primer can be viewed as a box query having one or more (in case of degenerate primers) characters along each dimension. Further, techniques such as DNA synthesis or PCR (Polymerase Chain Reaction) need two primers to define the region of the sequence that is to be processed (e.g., amplifies in case of PCR). The two candidate primers can be combined together to form a larger box query which can accelerate the search. In this paper we present performance of Bond-tree in primer design applications.

Rest of the paper is organized as follows. We present 
relevant work in this area in the next section. Section 3 introduces the relevant concepts and notations used for our indexing scheme in the NDDS. Section 4 introduces the new heuristics to support efficient box queries in the NDDS based on our theoretical analysis. Section 5 presents the BoNDtree, including its tree structure, construction algorithms and relevant operations. Section 6 describes further improvement of the BoND-tree performance based on the compression of index nodes. Section 7 reports our experimental results. Concluding remarks follow in the last section.

\section{Related Work}

Many indexing schemes have been proposed for the CDS. Some well-known CDS indexing structures are the K-D-B tree [22], the R-tree [13], the $\mathrm{R}^{*}$-tree [2], the X-tree [4] and the LSDh-tree [14]. Indexing multi-dimensional vectors in the NDDS is a relatively new problem.

Traditional string indexing techniques such as Tries [9] and its derivatives (e.g., the suffix tree [27] and the ternary search tree [3], [9]) could be applied to discrete data when the vectors to be indexed could be treated as strings. However, they are in-memory indexing structures which could not be utilized to support large scale data sets. There exist disk-based string indexing structures such as the prefix B-tree [1] and String B-tree [12] but they rely on the fact that indexed strings could be sorted - a property that does not exists in the NDDS.

The vantage-point tree [15], [29] and its variants like the MVP tree [5] are indexing techniques designed for the metric space [7]. As a special case of the metric space, the vector space [25], [28] including NDDSs could also be indexed by metric indexing structures. But a major drawback of these techniques is that they are static main memory-based structures which focus on reducing the number of distance computations. As a dynamic metric space indexing structure designed for large scale databases, the M-tree [8] is another indexing approach which could be applied to NDDSs. However, it could only use the relevant distance between vectors when creating the indexing structure. The special characteristics of the NDDS such as occurrences and distributions of data points on each dimension are totally ignored by the M-tree (as well as other metric space indexing methods), which could affect its retrieval performance when compared to indexing techniques designed specifically for the NDDS. It has been shown that when retrieving data for box queries the M-tree performance is significantly worse than that of the ND-tree [20], a technique recently proposed to support efficient indexing of the NDDS.

De Vries et al. [10] propose an interesting data decomposition technique for $\mathrm{k}-\mathrm{NN}$ search in real valued data. They divide the indexed dimensions vertically to create slices of dimensions. Then each slice is stored sequentially. For k-NN queries, observing only first few dimensions provides enough information to prune most of the data records. Hence, despite lack of any conventional indexing structure, this method provides good performance for high dimensional data. However, indexing techniques that work well for similarity queries do not necessarily support box(window) queries efficiently. This is because query conditions for box queries are specified for each dimension separately - any indexed vector which has conflicts with the query condition on any dimension is pruned away immediately from the result set. On the other hand, similarity queries are interested in vectors similar to the given query vector. The concept of similarity (or dissimilarity) between vectors are calculated based on the information combined from all dimensions. As a result, when organizing vectors in an indexing structure, heuristics efficient for similarity queries cannot guarantee good performance for box queries. In fact, in this paper we propose two new heuristics for distributing indexed vectors in a new index tree, i.e. the BoND-tree, to support efficient box queries. Although the two new heuristics may not be intuitive at a first glance, both our theoretical analysis and experimental results demonstrate that they are very effective in supporting box queries in the NDDS. We also show that for a real world application of primer design for genome sequence database, our proposed scheme can be applied with a significant improvement in performance.

\section{BASIC CONCEPTS}

In this section we introduce critical geometric concepts extended from the CDS to the NDDS. Like the indexing techniques in [20] and [21], our new BoND-tree uses these geometric concepts to optimize the organization of indexed vectors during its construction time.

A Non-ordered Discrete Data Space $\Omega_{d}$ is a multidimensional vector data space, where $d$ is the total number of dimensions in $\Omega_{d}$. Each dimension in $\Omega_{d}$ has an alphabet $A_{i}(1 \leq i \leq d)$ consisting of a finite number of characters, where no natural ordering exists among the characters.

A rectangle $R$ in $\Omega_{d}$ is defined as $R=S_{1} \times S_{2} \times S_{3} \ldots \times$ $S_{d}$, where $S_{i} \subseteq A_{i} . S_{i}$ is called the $i$-th component set of $R$. The edge length of $R$ along dimension $i$ is defined as $\left|S_{i}\right|$, which is the cardinality of set $S_{i}$. If $\forall i \in\{1,2, \ldots, d\},\left|S_{i}\right|=$ $1, R$ degrades to a vector in $\Omega_{d}$. The area of a rectangle $R$ is defined as $R=\prod_{i=1}^{d}\left|S_{i}\right|$. The overlap of a set of rectangles is defined as the Cartesian product of the intersections of all the rectangles' component sets on each dimension.

Given a set of rectangles $S R=\left\{R_{1}, R_{2}, \ldots, R_{j}\right\}$, if $\forall i \in\{1,2, \ldots, d\}$ and $\forall t \in\{1,2, \ldots, j\}$, the $i$-th component set of a rectangle $R$ contains the $i$-th component set of $R_{t}, \mathrm{R}$ is a discrete bounding rectangle of $S R$. A discrete minimum bounding rectangle (DMBR) of $S R$ is such a discrete bounding rectangle that has the least area among all the discrete bounding rectangles of $S R$. The span of a DMBR $R$ along dimension $i$ is defined as the edge length of $R$ along dimension $i$.

In order to control the contribution of each dimension in the geometric concepts such as the area, a normalization is applied (i.e., the edge length of each dimension is normalized by the domain size of the corresponding dimension). Detailed definition and explanation of these concepts could be found in [21].

\section{Optimization of INDEX Trees for BoX QUERIES IN THE NDDS}

We start by discussing box queries in the NDDS in section 4.1. In section 4.2 we present a method to calculate estimated 


\begin{tabular}{|l|l|}
\hline Symbol & Explanation \\
\hline$d$ & Number of dimensions \\
\hline$\Omega_{d}$ & $d$-dimensional NDDS \\
\hline$A_{i}$ & Alphabet size of the $i^{t h}$ dimension \\
\hline$R$ & Rectangle in $\Omega_{d}$ \\
\hline$D_{i}$ & Component of $R$ along the $i^{\text {th }}$ dimension \\
\hline$S R$ & Set of rectangles.in $\Omega_{d}$ \\
\hline$q$ & A fixed box query \\
\hline$Q$ & Random box query in $\Omega_{d}$ \\
\hline$w$ & Query window of $q$ \\
\hline$W$ & Query window of $Q$ \\
\hline
\end{tabular}

TABLE 1: Table of important symbols used in the paper box query I/O for hierarchical indexing structures. In section 4.3 we discuss the splitting problem of index trees and show that box queries require specifically designed heuristics when building a tree. New heuristics to support efficient box queries in the NDDS are introduced in section 4.4.

\subsection{Box Queries in the NDDS}

A box query $q$ on a data set in an NDDS is a query which is specified by listing the set of values that each dimension is allowed to take. More formally, given an NDDS $\Omega_{d}$, suppose $q c_{i} \subseteq A_{i}\left(A_{i}\right.$ is the alphabet of $\Omega_{d}$ on dimension $\left.i, 1 \leq i \leq d\right)$ is the set of values allowed by a box query $q$ along dimension $i$, we use $w=\prod_{i=1}^{d} q c_{i}$ to represent the query window of box query $q$. Any vector $V=\left(v_{1}, v_{2}, \ldots, v_{d}\right)$ inside $w$ (i.e., $\left.v_{i} \in q c_{i}, \forall i \in\{1,2, \ldots, d\}\right)$ is returned in the result of the box query $q$.

Given a hierarchical indexing structure, suppose $F(N, q)$ is a boolean function which returns true when and only when the query window of a box query $q$ overlaps with the DMBR of a node $N$ in an index tree, box query $q$ is typically evaluated as follows: starting from the root node $R$ (let $N=R$ ), the query window of $q$ is compared with the DMBRs of all the child nodes of $N$. Any child node $N^{\prime}$ for which $F\left(N^{\prime}, q\right)=1$ is recursively evaluated using the same procedure. However, if $q$ does not overlap with a child node $N^{\prime \prime}$ (i.e., $F\left(N^{\prime \prime}, q\right)=0$ ), $N^{\prime \prime}$ and its child nodes can be pruned from the search path. Assuming each node occupies one disk block, the query I/O is the total number of nodes accessed during the query process.

In section 4.2 we show how to estimate box query I/O for an index tree in the NDDS.

\subsection{Expected I/O for Box Queries}

From the generic query execution procedure described in the previous section, it is clear that a node $N$ needs to be accessed (and thus contributes to the query $\mathrm{I} / \mathrm{O}$ ) if and only if its DMBR overlaps with the query window $w$ of the box query $q$. Hence we have the following proposition:

Proposition 1: The number of I/O for evaluating a box query $q$ with query window $w$ using an index tree $T$ is given by:

$$
I O(T, q)=\sum_{N \text { is }} O(N, w),
$$

where

$$
O(N, w)= \begin{cases}1 & \text { if } w \text { overlaps with DMBR of } \mathrm{N} \\ 0 & \text { otherwise }\end{cases}
$$

Proof: Note that, execution of a box query will access every node whose DMBR overlaps with the query window. As each node access in the index tree results in one page access, the total number of I/O for the query is equal to the number of the overlapping nodes. Hence the result.

Note that proposition 1 is applied to a given (fixed) box query $q$ with query window $w$. However, in practice, we are more interested in the average performance of an indexing structure when answering a large number of box queries. More specifically, we need a way to evaluate an indexing structure $T$ 's average performance on supporting a query class $Q$ in an NDDS $\Omega_{d}$. Here we use a query class to represent a class of fixed box queries whose query windows have the same edge length on every dimension in $\Omega_{d}$. A query class $Q$ in $\Omega_{d}$ is defined as follows:

$$
\begin{aligned}
Q= & \left\{q_{\delta} \mid 1 \leq \delta \leq n ; \forall i, j \in\{1,2, \ldots, n\}, \forall k \in\{1,2, \ldots,\right. \\
& d\}, w_{i} \text { and } w_{j} \text { have the same edge length on }
\end{aligned}
$$

dimension $k$, where $w_{\delta}$ is the query window of box query $\left.q_{\delta}\right\}$.

For simplicity, in the rest of this paper, we call $Q$ a random box query which has query window $W$ (in contrast to a fixed box query $q$ with query window $w$ ) in a given NDDS. We use $w$ to represent a fixed query window which specifies the exact characters occurred on each dimension of an NDDS. A query window $W$ is used only to specify the number of characters on every dimension for a random box query $Q$.

Consider an index tree $T$ built in a $d$-dimensional NDDS $\Omega_{d}=A_{1} \times A_{2} \times \ldots \times A_{d}$. Suppose a node $N$ in $T$ has DMBR $R=S_{1} \times S_{2} \times \ldots \times S_{d}$ and $\left|S_{i}\right|=m_{i}\left(S_{i} \subseteq A_{i}, 1 \leq i \leq\right.$ d). For any box query $Q$ with query window $W$, if $W$ has $b_{i}\left(b_{i} \leq\left|A_{i}\right|\right)$ characters along dimension $i$, the probability of $R$ overlapping with $W$ along dimension $i$ is:

$$
O_{p, i}(N, W)=1-\frac{C_{\left|A_{i}\right|-m_{i}}^{b_{i}}}{C_{\left|A_{i}\right|}^{b_{i}}}, \quad(1 \leq i \leq d)
$$

Here we use the notation $C_{n}^{k}$ to denote the number of combinations of $n$ objects taken $k$ at a time. From formula (1), the probability for a node $N$ to overlap with a query window $W$ on all dimensions is calculated as follows.

$$
O_{p}(N, W)=\prod_{i=1}^{d}\left(1-\frac{C_{\left|A_{i}\right|-m_{i}}^{b_{i}}}{C_{\left|A_{i}\right|}^{b_{i}}}\right)
$$

Formula (2) gives the overlapping probability between a node $N$ 's DMBR and a query window $W$. Clearly, the overlapping probability is inversely proportional to the filtering power (pruning power) of $N$. In the rest of this paper we use the term filtering power to describe the chance that $N$ is pruned away from the query path when executing a box query $Q$.

We have the following proposition to estimate the average query $\mathrm{I} / \mathrm{O}$ of an index tree $T$ for a box query $Q$.

Proposition 2: The average (expected) I/O of executing a random box query $Q$ with query window $W$ for an index tree 
$T$ can be calculated as,

$$
I O(T, Q)=\sum_{N \text { is } T^{\prime} \text { s node }} O_{p}(N, W)
$$

Proof: The expected number of I/O for a random query $Q$ can be calculated as,

$$
\begin{aligned}
I O(T, Q) & =\sum_{N \text { is }} \begin{array}{l}
O_{p}(N, W) \times \\
\mathrm{I} / \mathrm{O} \text { required for accessing } \mathrm{N}
\end{array} \\
& =\sum_{N \text { is } T^{\prime} s \text { node }} O_{p}(N, W) \times 1 \\
& =\sum_{N \text { is } T_{T^{\prime}} \text { s node }} O_{p}(N, W)
\end{aligned}
$$

The theoretical analysis in the following subsections uses Proposition 2 to estimate performance of indexing structures for box queries in the NDDS.

\subsection{A Motivating Example for the Splitting Heuristics}

When using a tree structure for indexing data, the algorithms used for splitting overflow nodes play an important role in determining the index tree's query performance. This is because except the first node (which is created by default) in the tree, every other node is created by splitting an existing node. In order to reduce query $\mathrm{I} / \mathrm{O}$ for box queries in the NDDS, we want a splitting algorithm which distributes an overflow node's entries into the two new nodes in such a way that the resulting indexing structure will have minimum expected box query I/O in the NDDS. The expected number of I/O is given by Proposition 2.

Note that here we are interested in a splitting algorithm designed for random box queries rather than a particular box query. This is because we cannot make any assumption about the box queries which will be performed on the indexing structure. On the other hand, like other existing indexing techniques (e.g., the R-tree, the $\mathrm{R}^{*}$-tree, the $\mathrm{ND}$-tree, etc.), our splitting algorithm optimizes the indexing structure only based on the information available at the splitting time. That is, we do not make assumption about vectors which will be indexed after the splitting.

One of the recently proposed indexing schemes for supporting similarity searches in the NDDS is the ND-tree [20]. It adopts four heuristics for node splitting, which are: (1) $\mathrm{SH}_{1}$ Minimize Overlap (minimize the overlap between DMBRs of the new nodes), (2) $\mathrm{SH}_{2}$-Maximize Span (split along the dimension with the maximum edge length), (3) $\mathrm{SH}_{3}$-Center Split (balance the edge lengths of new nodes along the splitting dimension) and (4) $\mathrm{SH}_{4}$-Minimize Area (minimize the total area of the new nodes' DMBRs).

Our analysis of box queries in the NDDS suggest that although the minimize overlap heuristic is important for supporting efficient box queries, the others may not be. We illustrate this by the following example.

Consider a dimension $i$ with alphabet $\{a, b, c, \ldots, h\}$ (note the characters in the alphabet are non-ordered). Let $N$ be a node with characters $\{a, b, c, d\}$ along dimension $i$ in its DMBR. Consider two candidate partitions of $N$ : the first candidate partition $C P_{1}$ splits $N$ into two new nodes $N_{1}$ and $N_{2}$ with $\{a\}$ and $\{b, c, d\}$ on the $i$-th dimension in their respective DMBRs, and the second candidate partition $C P_{2}$ splits $N$ into nodes $N_{1}^{\prime}$ and $N_{2}^{\prime}$ with $\{a, c\}$ and $\{b, d\}$ along dimension $i$ in their respective DMBRs. Further, suppose we are considering a random box query $Q$ whose query window $W$ has 3 characters along dimension $i$. From formula (1), the probabilities of overlapping with the query window $W$ on the $i$-th dimension is 0.375 for node $N_{1}$ and 0.821 for node $N_{2}$, respectively. Similarly the probabilities of overlapping with $W$ on the $i$-th dimension are 0.643 and 0.643 for $N_{1}^{\prime}$ and $N_{2}^{\prime}$, respectively. Since $0.375+0.821<0.643+0.643=1.286$, when answering a random box query $Q, C P_{1}$ gives better filtering power on dimension $i$ than $C P_{2}$ (because $N_{1}$ and $N_{2}$ has less chance of overlapping with the query window on dimension $i$ than $N_{1}^{\prime}$ and $N_{2}^{\prime}$ ).

However, the ND-tree splitting algorithm would prefer the candidate partition $C P_{2}$ over $C P_{1}$ based on its heuristic $\mathrm{SH}_{3}$. This suggests that there exist better ways of splitting a dimension for box queries in the NDDS. Similarly, we can also come up with examples showing that splitting an overflow node on the dimension with a shorter span (edge length) can result in better filtering power (i.e., less probability of overlapping with the query window) than splitting the dimension with the maximum span (i.e., $\mathrm{SH}_{2}$ ).

In the following subsection we introduce the theoretical bases for the heuristics to be used in the proposed BoNDtree to support efficient box queries in the NDDS based on our theoretical analysis.

\subsection{Theoretical Basis for Node Splitting Heuristics}

When distributing vectors in an overflow node into two new nodes, we try to obtain overlap-free partitions in order to minimize the chance of searching both paths at query time. Unlike in the CDS, more overlap-free partitions are available in the NDDS due to the fact that elements in the NDDS are non-ordered and discrete. In this section we introduce two new heuristics for choosing overlap-free partitions of an overflow node $N$ of an index tree in the NDDS.

For the purpose of simplicity, we assume the NDDS to be indexed has the same alphabet size for each dimension and consider box queries which are uniform. A random box query $Q$ is said to be uniform if the edge lengths of the query window are the same along all dimensions. The common edge length is said to be the box size of the uniform box query $Q$. In fact, the theoretical analysis provided here could be extended to more complex situations where box queries are not uniform.

Consider a $d$-dimensional NDDS $\Omega_{d}$, an overflow node $N$, and a splitting dimension $u$ with edge length $x$. Consider two candidate partitions $C P_{1}$ and $C P_{2}$ along $u$ : $C P_{1}$ distributes the entries in $N$ between two new nodes $N_{1}$ and $N_{2}$; similarly $\mathrm{CP}_{2}$ splits $N$ into two new nodes $N_{1}^{\prime}$ and $N_{2}^{\prime}$. Suppose the edge lengths on dimension $u$ is $l$ in $N_{1}$ 's DMBR and it is $x-l$ in $N_{2}$ 's DMBR. And suppose the edge lengths on dimension $u$ in the DMBRs of $N_{1}^{\prime}$ and $N_{2}^{\prime}$ are $t$ and $x-t$, respectively. Here we assume $l<x-l$ and $t<x-t$. The filtering powers of the new nodes generated from $C P_{1}$ and $C P_{2}$ could be evaluated using the following theorem. 
Theorem 1: For the given splitting dimension $u$, if $l<t$, the probability of overlapping between the query window $W$ of a uniform box query $Q$ and DMBRs of $N_{1}$ and $N_{2}$ is smaller than the probability of overlapping between $W$ and the DMBRs of $N_{1}^{\prime}$ and $N_{2}^{\prime}$.

Proof: For any node with edge length $x$ and query window with edge length $b$ on dimension $u$, the probability of nodes $N_{1}$ and $N_{2}$ not overlapping with the query window on $u$ is $P_{1}=\frac{C_{A-x+l}^{b}}{C^{b}}+\frac{C_{A-l}^{b}}{C_{A}^{b}}$, where $A$ is the domain size of dimension $u$. Similarly, we have the non-overlapping probability of $N_{1}^{\prime}$ and $N_{2}^{\prime}$ with the query window as $P_{2}=\frac{C_{A-x+t}^{b}}{C_{A}^{b}}+\frac{C_{A-t}^{b}}{C_{A}^{b}}$. Thus, we want to show $P_{1} \geq P_{2}$, which equals to:

$$
C_{A-x+l}^{b}+C_{A-l}^{b} \geq C_{A-x+t}^{b}+C_{A-t}^{b} \quad(l<t)
$$

Let $\alpha=A-x+t, \beta=A-x+l$ and $\delta=x-l-t$. Then formula (3) simplifies to,

$$
C_{\alpha+\delta}^{b}-C_{\beta+\delta}^{b} \geq C_{\alpha}^{b}-C_{\beta}^{b}
$$

Using mathematical induction on $b$, when $b=1$, inequality (4) holds. Suppose it holds when $b=b^{\prime}$. Since $C_{m}^{n+1}=$ $\frac{m-n}{n+1} C_{m}^{n}$, when $b=b^{\prime}+1$, formula (4) becomes

$$
C_{\alpha+\delta}^{b^{\prime}} \frac{\alpha+\delta-b^{\prime}}{b^{\prime}+1}-C_{\beta+\delta}^{b^{\prime}} \frac{\delta}{b^{\prime}+1} \geq C_{\alpha}^{b^{\prime}} \frac{\alpha-b^{\prime}}{b^{\prime}+1}
$$

Since $C_{\alpha+\delta}^{b^{\prime}}>C_{\beta+\delta}^{b^{\prime}}$,

$$
\begin{aligned}
& C_{\alpha+\delta}^{b^{\prime}} \frac{\alpha+\delta-b^{\prime}}{b^{\prime}+1}-C_{\beta+\delta}^{b^{\prime}} \frac{\delta}{b^{\prime}+1} \geq C_{\beta+\delta}^{b^{\prime}} \frac{\alpha+\delta-b^{\prime}}{b^{\prime}+1}- \\
& C_{\beta+\delta}^{b^{\prime}} \frac{\delta}{b^{\prime}+1}=C_{\beta+\delta}^{b^{\prime}} \frac{\alpha-b^{\prime}}{b^{\prime}+1} \geq C_{\alpha}^{b^{\prime}} \frac{\alpha-b^{\prime}}{b^{\prime}+1}
\end{aligned}
$$

Inequality (5) shows the correctness of Theorem 1 for uniform box queries. The following corollary proves that theorem holds even for non-uniform box queries.

Corollary 1: For the given splitting dimension $u$, if $l<t$, the probability of overlapping between the query window $W$ of a non-uniform box query $Q$ and DMBRs of $N_{1}$ and $N_{2}$ is smaller than the probability of overlapping between $W$ and the DMBRs of $N_{1}^{\prime}$ and $N_{2}^{\prime}$.

Proof: For any dimension $1 \leq u \leq d$, we want to show:

$$
\sum_{i=1}^{s}\left(C_{A-x+l}^{b_{i u}}+C_{A-l}^{b_{i u}}\right) \geq \sum_{i=1}^{s}\left(C_{A-x+t}^{b_{i u}}+C_{A-t}^{b_{i u}}\right) \quad(l \leq t)
$$

We have already proved that inequality (3) holds. Thus we know given $b_{i} u(1 \leq i \leq u)$, inequality

$$
\left(C_{A-x+l}^{b_{i u}}+C_{A-l}^{b_{i u}}\right) \geq\left(C_{A-x+t}^{b_{i u}}+C_{A-t}^{b_{i u}}\right) \quad(l \leq t)
$$

holds. Substitution of (7) into inequality (6) proves the correctness of inequality (6).

Theorem 1 suggests splitting an overflow node by putting as many characters as possible into one new node on the splitting dimension. This is contrary to heuristic $\mathrm{SH}_{3}$ used by the ND-tree. Note that a data-partitioning based index tree has a minimum utilization criterion, which enforces that a certain percentage of the disk block for a tree node should always be filled. When applying Theorem 1, the minimum utilization criterion needs to be considered. This means that the most unbalanced candidate partition which satisfies the minimum utilization criterion should be selected because it has the least overlapping probability (among all candidate partitions generated from a splitting dimension $u$ which satisfy the minimum utilization criterion) based on Theorem 1 .

We use the following theorem to choose splitting dimensions for box queries in the NDDS:

Theorem 2: Given an overflow node $N$ and a uniform box query (i.e. all the sides of the box have the same length) $Q$ with query window $W$, splitting $N$ on a dimension $u$ in $\left\{u \mid E L_{u}>\right.$ $1 ;$ for any $1 \leq i \leq d$, either $E L_{i} \geq E L_{u}$ or $\left.E L_{i}=1\right\}$ gives less probability of overlap between $W$ and the DMBRs of the two newly created nodes than splitting $N$ on other dimensions, where $E L_{i}(1 \leq i \leq d)$ is the edge length of $N$ 's DMBR along dimension $i$

Proof: First we show that, when supporting uniform box queries, splitting a node on a dimension $p$ with edge length $x$ gives more filtering power than splitting on dimension $q$ with edge length $x+1$. From Theorem 1, we know that the best way to split a dimension is the most unbalanced split. Suppose that both dimensions have alphabet size $A$, when splitting the dimension with edge length $x$, the overlapping probability is calculated as:

$$
\left(1-\frac{C_{A-1}^{b}}{C_{A}^{b}}+1-\frac{C_{A-(x-1)}^{b}}{C_{A}^{b}}\right)\left(1-\frac{C_{A-(x+1)}^{b}}{C_{A}^{b}}\right)
$$

Similarly, the overlapping probability when splitting the dimension with edge length $x+1$ is

$$
\left(1-\frac{C_{A-1}^{b}}{C_{A}^{b}}+1-\frac{C_{A-x}^{b}}{C_{A}^{b}}\right)\left(1-\frac{C_{A-x}^{b}}{C_{A}^{b}}\right)
$$

Substituting $C_{A-x+1}^{b}=\frac{A-x+1}{A-x+1-b} C_{A-x}^{b} \quad$ and $C_{A-x-1}^{b}=\frac{A-x-b}{A-x} C_{A-x}^{b}$ into expressions 8 and 9 , and noting that $C_{A-1}^{b}=\left(1-\frac{b}{A}\right) C_{A}^{b}$, we need to prove that,

$$
\frac{\left(A-b x+b-b^{2}\right)}{A} \leq \frac{C_{A-x}^{b}}{C_{A}^{b}}
$$

Using mathematical induction on $b$, formula (10) holds when $b=1$. Suppose it holds when $b=b^{\prime}$.

Let $\frac{\left(A-b^{\prime} x+b^{\prime}-b^{\prime^{2}}\right)}{A}=\alpha, \frac{C_{A-x}^{b^{\prime}}}{C_{A}^{b^{\prime}}}=\beta$, we know that $\alpha \leq \beta$. When $b=b^{\prime}+1$, the left side of (10) becomes

$$
\frac{A-b^{\prime} x-x+b^{\prime}+1-b^{\prime^{2}}-2 b^{\prime}-1}{A}=\alpha-\frac{x+2 b^{\prime}}{A}
$$

and the right side of formula (10) becomes

$$
\frac{C_{A-x}^{b^{\prime}+1}}{C_{A}^{b^{\prime}+1}}=\frac{C_{A-x}^{b^{\prime}}}{C_{A}^{b^{\prime}}} \frac{\frac{A-x-b^{\prime}}{b^{\prime}+1}}{\frac{A-b^{\prime}}{b^{\prime}+1}}=\beta\left(1-\frac{x}{A-b^{\prime}}\right)
$$

So we want to show that

$$
\alpha-\frac{x+2 b^{\prime}}{A} \leq \beta\left(1-\frac{x}{A-b^{\prime}}\right)
$$


Since $\alpha \leq \beta$, we only need to show:

$$
\begin{gathered}
\frac{x+2 b^{\prime}}{A} \geq \beta \frac{x}{A-b^{\prime}} \\
\Longleftrightarrow \\
\frac{x+2 b^{\prime}}{x} \geq \frac{(A-x)(A-x-1) \ldots\left(A-x-b^{\prime}+1\right)}{(A-1) \ldots\left(A-b^{\prime}\right)}
\end{gathered}
$$

Left side of (13) has

$$
\frac{x+2 b^{\prime}}{x}=1+\frac{2 b^{\prime}}{x} \geq 1
$$

On the right side of (13), since $x>1$, we have

$$
\frac{(A-x)(A-x-1) \ldots\left(A-x-b^{\prime}+1\right)}{(A-1) \ldots\left(A-b^{\prime}\right)}<1
$$

Thus we know (13) holds, which shows that splitting on dimension $p$ with length $x$ gives better filtering power than splitting on dimension $q$ with length $x+1$ for fixed query box sizes. It is straightforward to deduce that for any $n \geq 1$, dimension with length $x$ will give better splitting than dimension with length $x+n$.

Theorem 2 strictly applies to uniform box queries. The following corollary proves that the theorem also holds for a non-uniform box queries.

Corollary 2: Given an overflow node $N$ and a non-uniform box query $Q$ with query window $W$, splitting $N$ on a dimension $u$ in $\left\{u \mid E L_{u}>1 ;\right.$ for any $1 \leq i \leq d$, either $E L_{i} \geq$ $E L_{u}$ or $\left.E L_{i}=1\right\}$ gives less probability of overlap between $W$ and the DMBRs of the two newly created nodes than splitting $N$ on other dimensions.

Proof: Consider a query box $Q_{i}(1 \leq i \leq s)$, Overlapping probability when splitting dimension $p$ is

$$
\left(1-\frac{C_{A-1}^{b_{i} p}}{C_{A}^{b_{i} p}}+1-\frac{C_{A-(x-1)}^{b_{i} p}}{C_{A}^{b_{i} p}}\right)\left(1-\frac{C_{A-(x+1)}^{b_{i} p}}{C_{A}^{b_{i} p}}\right)
$$

Overlapping probability when splitting dimension $q$ is

$$
\left(1-\frac{C_{A-1}^{b_{i} q}}{C_{A}^{b_{i} q}}+1-\frac{C_{A-x}^{b_{i} q}}{C_{A}^{b_{i} q}}\right)\left(1-\frac{C_{A-x}^{b_{i} q}}{C_{A}^{b_{i} q}}\right)
$$

When the edge lengths of $Q_{1} \sim Q_{s}$ are uniformly distributed within $\left[t_{1}, t_{r}\right]$, formulas (14) and (15) could be rewritten as,

$$
\gamma \sum_{j=1}^{r}\left(1-\frac{C_{A-1}^{t_{j}}}{C_{A}^{t_{j}}}+1-\frac{C_{A-(x-1)}^{t_{j}}}{C_{A}^{t_{j}}}\right)\left(1-\frac{C_{A-(x+1)}^{t_{j}}}{C_{A}^{t_{j}}}\right)
$$

and

$$
\gamma \sum_{i=1}^{s}\left(1-\frac{C_{A-1}^{t_{j}}}{C_{A}^{t_{j}}}+1-\frac{C_{A-x}^{t_{j}}}{C_{A}^{t_{j}}}\right)\left(1-\frac{C_{A-x}^{t_{j}}}{C_{A}^{t_{j}}}\right)
$$

correspondingly, where $\gamma$ is a constant factor.

We need to show that the value of expression 16 is less than or equal to the value of expression 17. But as a part of the proof of theorem 2, we have already shown that individual terms of the summation obey the inequality (i.e. value of the expression 8 is less than or equal to the value of expression 9). Hence, the summation must obey the inequality. This proves the corollary for non-uniform query boxes.
Theorem 2 suggests splitting an overflow node along a dimension which has a shorter edge length in the node's DMBR. This is opposite of heuristic $\mathrm{SH}_{2}$ used by the ND-tree splitting algorithm. Again we see that, to support box queries in the NDDS, there could be better ways to select splitting dimensions compared to the heuristics used by the ND-tree.

\subsection{Splitting Heuristics}

Given theorems 1 and 2, we propose the following heuristics for splitting an overflow node in the NDDS. The heuristics are applied in the order they are specified.

\section{R1: Minimum Overlap}

Of all the candidate partitions, heuristic R1 selects the one that results in the minimum overlap between the DMBRs of the newly created nodes. This heuristic is the same as the one used by some of the existing works [2], [20].

\section{R2: Minimum Span}

If R1 generates more than one overlap-free partitions, heuristic $\mathrm{R} 2$ selects one of those partitions which is generated from splitting a dimension with the smallest span. This follows directly from theorem 2 .

\section{R3: Minimum Balance}

Given a splitting dimension $u$, heuristic R3 chooses the most unbalanced overlap-free partition (i.e., the one that puts as few characters as possible in one node's DMBR and as many characters as possible in the other node's DMBR on dimension $u$ ) among all candidate partitions which satisfy the minimum utilization criterion and tied on R2. This follows directly from theorem 1.

It is possible that, even after applying all the heuristics, there remain more than one candidate partition. In such cases a partition is chosen randomly from the tied ones.

Heuristics R2 and R3 may not be intuitive at a first glance (e.g. the binary search has been proved to be an efficient searching algorithm in the CDS, which implies a balanced partition of the indexed data space). But these heuristics try to exploit the properties pertinent to box queries in the NDDS. It is the nature of the data space that makes seemingly unintuitive splitting heuristics perform better than the ones used in the CDS. We will see the experimental results in section 7 .

\section{Construction of the Bond-tree}

In this section, we describe the data structure and important algorithms for constructing the proposed BoND-tree.

\subsection{Insertion procedure}

A BoND-tree is a balanced indexing structure which has the following properties: (1) Each tree node occupies one disk block; (2) All nodes must have at least a given minimum amount of space filled by indexed entries unless it is the root node (the minimum space utilization requirement); (3) The root node has at least 2 indexed entries unless it is a leaf node; (4) A leaf node entry structure has the form $(V, P)$, where $V$ is an indexed vector (key) and $P$ is the pointer to the relevant tuple in the database corresponding to $V$; (5) A non-leaf node entry structure has the form $(D, P)$, where $D$ 
is the DMBR of the entry's corresponding child node and $P$ is the pointer to that child node.

We use a bitmap structure to represent DMBR information in a non-leaf node entry. The overall data structure of the BoND-tree is inspired by that of the ND-tree. It is further optimized through the compressed BoND-tree introduced in section 6 .

Inserting a vector in the BoND-tree involves two steps. First, we find a suitable leaf node $L$ for the new vector. Then we put the vector into $L$ and update $L$ 's ancestor nodes' DMBRs as needed. The second step may cause a split of the leaf node (when an overflow occurs), which might trigger cascaded splits all the way to the root node.

\subsubsection{Selecting a Leaf Node}

Given a node $N$, the BoND-tree uses a select-node algorithm to pick an appropriate child node of $N$ which will accommodate a new vector $V$. If there is only one child node whose DMBR containing $V$, that node will be chosen to insert $V$. In case $V$ is covered by more than one child nodes' DMBRs, the node whose DMBR size is the smallest is selected. If $V$ is covered by $N$ 's DMBR but not covered by any of $N$ 's child nodes' DMBRs, we use the 3 heuristics proposed by the ND-tree [20] for selecting a child node, which are: Minimum Overlap Enlargement, Minimum Area Enlargement, and Minimum Area. The heuristics are applied in the order they are presented. That is, a heuristic will be used if and only if application of the previous heuristic(s) results in one or more ties.

To insert a new vector into the BoND-tree, we need to find a leaf node to accommodate the vector. This is achieved by invoking the select-node algorithm recursively, starting from the root node of the tree, until a leaf node is selected.

\subsubsection{Splitting an Overflow Node}

As discussed in section 4.4, a better way to split an overflowing node $N$ in the NDDS is to get an overlap-free and unbalanced split along a dimension $i$, which has the minimum span among all dimensions whose spans are larger than 1. Among the heuristics suggested in section 4.4, R2 could be achieved by comparing the span of each dimension in node $N$ 's DMBR. However, implementation of R3 in the BoND-tree is more complex, especially at the non-leaf levels of the tree. This is because the component sets of the DMBRs of non-leaf node entries could have more than one character on a dimension. Table 2 shows an example of different $i$-th component sets from 8 non-leaf node entries $\left(E_{1}, E_{2}, \ldots, E_{8}\right)$ on a dimension $i$ which has the alphabet $\{a, b, c, d, e, f, g\}$.

\begin{tabular}{l|cccc}
\hline Non-leaf entry & $E_{1}$ & $E_{2}$ & $E_{3}$ & $E_{4}$ \\
\hline Component set & $\{a, b\}$ & $\{b, c\}$ & $\{a, c\}$ & $\{a, b, c\}$ \\
\hline \hline Non-leaf entry & $E_{5}$ & $E_{6}$ & $E_{7}$ & $E_{8}$ \\
\hline Component set & $\{a, b, e\}$ & $\{e\}$ & $\{e, f, g\}$ & $\{f\}$ \\
\hline
\end{tabular}

TABLE 2: Different component sets of non-leaf entries on dimension $i$.

When generating candidate partitions on dimension $i$, we could have a component set which is a proper subset of other sets like $\{e\}$ and $\{e, f, g\}$; sets which are disjoint or partly overlapped like $\{a, b\},\{e\}$ and $\{a, b, e\}$; sets whose union is only part of the alphabet or the whole alphabet such as $\{a, b, c\},\{f\}$ and $\{e, f, g\}$; or a single component set which contains all the characters from the alphabet. The relationship among component sets at a non-leaf level could be very complex in the NDDS.

\subsection{The Node Splitting Problem}

In this section we analyze how an overflow node $N$ is split in the BoND-tree using heuristic R3. Suppose $u$ is the dimension along which we will generate candidate partitions for $N$, we first group all entries which share common characters along dimension $u$ such that the $u$-th component sets of any two entries from different groups are disjoint. Each group is then treated as a single item when splitting the node. Grouping entries this way avoids distributing entries with the same character(s) along dimension $u$ into two different nodes (in which case an non-overlap-free partition is generated). Each group has a certain number of characters along dimension $u$ and requires a certain amount of space to store the entries in it. We use $G_{1}, G_{2}, \ldots, G_{n}$ to represent these groups.

Suppose $S_{d}$ is the disk block size occupied by each tree node and the minimum space utilization criterion requires that a certain size $S_{\text {min }}$ of each node must be filled. Based on our discussion, the BoND-tree node splitting problem using heuristic R3 could be defined as follows.

\section{Node Splitting Problem of the BoND-tree Using Heuris-} tic R3 (NSP): Given entry groups $G_{1}, G_{2}, \ldots, G_{n}$ in an overflow node $N$, suppose the number of characters (along the splitting dimension) and the storage space of each of the groups are $G V_{1}, G V_{2}, \ldots, G V_{n}$ and $G W_{1}, G W_{2}, \ldots, G W_{n}$ respectively. The BoND-tree splitting algorithm distributes the entry groups to two new nodes $N_{1}$ and $N_{2}$ such that,

(1) The total number of characters $V_{\text {total }}=\sum_{G_{i} \text { in } N_{1}} G V_{i}$ is

the maximum

$$
G_{i}
$$

(2) Both $N W_{1}=\sum_{G_{i}} G W_{i}$ and $N W_{2}=\sum_{G_{i}} G W_{i}$ satisfy the minimum space utilization criterion of the tree (i.e., $N W_{1} \geq S_{\min }$ and $\left.N W_{2} \geq S_{\min }\right)$.

One brute force way to solve problem NSP is to compute all permutations of the entry groups in an overflow node, and then put splitting points tentatively between adjacent groups in each permutation to generate candidate partitions. But this clearly demands a heavy computation overhead. Even for a small number of entry groups, it would be impractical to evaluate all permutations (e.g., for 10 entry groups, the number of candidate partitions would be more than one million). To solve the problem efficiently, we further analyze the node splitting problem as follows.

Suppose $S_{e}$ is the size of each node entry. The maximum storage space $S_{\max }$ that could be utilized by a new node is calculated as:

$$
S_{\max }=\left(\left\lfloor S_{d} / S_{e}\right\rfloor+1-\left\lceil S_{\min } / S_{e}\right\rceil\right) \times S_{e}
$$


For example, consider a node $N$ containing 4 entries and each entry using $S_{e}=90$ bytes, the total space occupied by these 4 entries is $90 \times 4=360$ bytes. Suppose the disk block size $S_{d}$ is 400 bytes, $N$ will overflow if the 5 -th entry is inserted into it. Further suppose the minimum utilization criterion specifies that at least 100 bytes of each node must be filled $\left(S_{\min }=100\right)$. If $N$ is split into two new nodes, each new node must have at least $\left\lceil S_{\min } / S_{e}\right\rceil=2$ entries distributed to it. As a result, each of the new nodes could have at most $\left\lfloor S_{d} / S_{e}\right\rfloor+1-\left\lceil S_{\min } / S_{e}\right\rceil=3$ entries after the splitting. Thus a new node could use at most $S_{\max }=3 \times S_{e}=270$ bytes to store index entries distributed to it.

Formula (18) gives the maximum amount of space which could be utilized in each of the newly generated nodes to store indexed entries (so the remaining entries will be put in the other node). From formula (18), we could get the following property of $S_{\max }$ :

$$
S_{\max } \leq\left(\left\lfloor S_{d} / S_{e}\right\rfloor+1\right) \times S_{e}-S_{\min }
$$

From formula (19), we know that $\left(\left\lfloor S_{d} / S_{e}\right\rfloor+1\right) \times S_{e}-$ $S_{\max } \geq S_{\min }$, which means by allowing one new node to use no more than $S_{\max }$ size of space for storing node entries, the other node is guaranteed to have at least $S_{\min }$ space filled by entries distributed to it.

Given the maximum space $S_{\max }$ defined in formula (18), we tackle the node splitting problem NSP in the following way.

When a node $N$ is split to nodes $N_{1}$ and $N_{2}$, the splitting algorithm tries to distribute as many entries as possible to $N_{1}$, but the maximum space utilized in $N_{1}$ is no more than $S_{\max }$. Suppose the spaces occupied by entries distributed to $N_{1}$ and $N_{2}$ are $S_{1}$ and $S_{2}$ respectively. Clearly $S_{1}$ is no less than $S_{2}$ (since the splitting algorithm tries to put more entries into $N_{1}$ ). We already know from formula (19) that $S_{2}$ is no smaller than $S_{\text {min }}$. Since $S_{1} \geq S_{2}, S_{1}$ will be no less than $S_{\text {min }}$ either.

Based on our analysis above, we provide an alternative definition of the node splitting problem using heuristic R3, which is equivalent to the previous problem NSP. Note that in both definitions we distribute entry groups instead of entries in order to get overlap-free partitions.

Redefined Node Splitting Problem of the BoND-tree Using Heuristic R3 (RNSP): Given entry groups $G_{1}, G_{2}, \ldots, G_{n}$ in an overflow node $N$, suppose the number of characters (along the splitting dimension) and the storage space of all groups are $G V_{1}, G V_{2}, \ldots, G V_{n}$ and $G W_{1}, G W_{2}, \ldots, G W_{n}$ respectively. The BoND-tree splitting algorithm distributes the entry groups to two new nodes $N_{1}$ and $N_{2}$ such that,

(1) The total number of characters $V_{\text {total }}=\sum_{G_{i}} G V_{i}$ is the maximum.

(2) The total storage space $W_{\text {total }}=\sum_{G_{i}} G W_{i}$ satisfies the constraint $W_{\text {total }} \leq S_{\max }$, where $S_{\max }$ is calculated from formula (18).

Note that in the definition of problem RNSP, we use the maximum space constraint $S_{\max }$ on a single node $N_{1}$ to guarantee the minimum space requirement on both nodes specified in problem NSP. Our discussion above has already shown that both the requirements on $N_{1}$ and $N_{2}$ defined in NSP will be satisfied by enforcing the maximum space constraint $S_{\max }$ on the node $N_{1}$.

The redefined splitting problem can be mapped to the 01 Knapsack problem if we consider each entry group as the objects to be filled in the knapsack and $S_{\max }$ as the knapsack capacity. This mapping greatly simplifies the solution for the splitting problem.

\subsection{The Node Splitting Algorithm}

As the node splitting problem is mapped to the 0-1 knapsack problem, a dynamic programming solution [16], [23] can be used to solve it optimally and efficiently. After the items (entry groups) to be put into the knapsack (node $N_{1}$ ) is decided, the remaining items (entry groups) are put into node $N_{2}$.

Algorithm 1 summarizes all the important steps involved in inserting a new entry into a tree node.

Algorithm 1: insert_entry $(N, E)$

Input: A node $N$ and an entry $E$ to be inserted in $N$.

Output: Modified tree structure that accommodates entry $E$. Method:

1. if $N$ has space for $E$

2. Insert $E$ in the list of entries in $N$

3. Update DMBR of $N$ 's parent node as needed

4. else // We need to split $N$

5. Record dimensions with span larger than 1 into a list $L$

6. Sort $L$ based on dimension span in ascending order

7. for every dimension $i$ in $L$ do

8. Group entries in $N$ based on their component sets on dimension $i$

9. Calculate each entry group's weight and value //mapped to the $0-1$ Knapsack Problem

10. if $N$ is a leaf node

11. Solve the special case of the $0-1$ knapsack problem using the greedy approach

12. else

13. Solve the $0-1$ knapsack problem

14. end if using dynamic programming

15. if a solution satisfying the minimum utilization criterion is found

16.

17.

18. return the solution end if

\section{end for}

19. if no solution that is overlap-free and satisfies the minimum utilization criterion could be found

20. Generate candidate partitions based on the descending order of $r_{i}$ and select a partition with the least overlap

21. return the solution

22. end if

\section{3. end if}

Mapping the splitting problem RNSP into the 0-1 Knapsack Problem not only provides an efficient way to find the most 
suitable partition for an overflow node, but also allows the freedom of using different ways to build the BoND-tree based on the particular requirement and purpose of indexing.

For example, when both the query performance and the time needed to construct the indexing structure are critical, parallel algorithms [11], [18] for the 0-1 knapsack problem could be applied to build the BoND-tree efficiently and quickly. On the other hand, when the BoND-tree is created as a temporary indexing structure, the query $\mathrm{I} / \mathrm{O}$ is usually not the only (or the most important) consideration: sometimes people want to build index trees quickly and discard them after performing a limited number of queries. In such cases, the BoND-tree could be generated using algorithms introduced in [17] and [24], which provide approximate solutions with guaranteed closeness to the optimal solution with much a less time complexity and system resource requirements.

We illustrate the BoND-tree splitting algorithm using an example as shown below.

Let the entries in an overflow non-leaf node be $E_{1} \ldots E_{12}$. Further, suppose DMBRs of these entries have the component sets along a splitting dimension $u$ as shown in table 3 . After the

\begin{tabular}{c|cccccc}
\hline Entry & $E_{1}$ & $E_{2}$ & $E_{3}$ & $E_{4}$ & $E_{5}$ & $E_{6}$ \\
\hline Component set & $\{a\}$ & $\{b\}$ & $\{a, b, c\}$ & $\{d\}$ & $\{e\}$ & $\{e, f\}$ \\
\hline \hline Entry & $E_{7}$ & $E_{8}$ & $E_{9}$ & $E_{10}$ & $E_{11}$ & $E_{12}$ \\
\hline Component set & $\{f\}$ & $\{h, i\}$ & $\{i\}$ & $\{j\}$ & $\{j\}$ & $\{k\}$ \\
\hline
\end{tabular}

TABLE 3: Different component sets for non-leaf entries $E_{1} \sim$ $E_{12}$.

grouping process we obtain the following 6 groups as shown in table 4 . Each group $G_{i}$ has a set of characters $G S_{i}$ on the

\begin{tabular}{c|ccc}
\hline Group & $G_{1}$ & $G_{2}$ & $G_{3}$ \\
\hline Entries & $\left\{E_{1}, E_{2}, E_{3}\right\}$ & $\left\{E_{4}\right\}$ & $\left\{E_{5}, E_{6}, E_{7}\right\}$ \\
\hline \hline Group & $G_{4}$ & $G_{5}$ & $G_{6}$ \\
\hline Entries & $\left\{E_{8}, E_{9}\right\}$ & $\left\{E_{10}, E_{11}\right\}$ & $\left\{E_{12}\right\}$ \\
\hline
\end{tabular}

TABLE 4: Grouping of non-leaf entries. splitting dimension (by applying the set union operation on the component sets of all group members' DMBRs on dimension $u$ ). Here we use $G V_{i}$ to represent the number of characters in $G S_{i}$. Also each group requires certain space to store the entries in it. Let the amount of space required for each entry be one unit and the capacity of the node be 11 units. Further suppose the minimum space utilization requires each new node must utilize at least 3 units. We use $G W_{i}$ to represent the space required by $G_{i}$. Table 5 shows the item weights and values of the 0-1 knapsack problem mapped from the node splitting problem. According to heuristic R3, after splitting

\begin{tabular}{c|cccccc}
\hline Item & $G_{1}$ & $G_{2}$ & $G_{3}$ & $G_{4}$ & $G_{5}$ & $G_{6}$ \\
\hline Weight & 3 & 1 & 3 & 2 & 2 & 1 \\
\hline Value & 3 & 1 & 2 & 2 & 1 & 1 \\
\hline
\end{tabular}

TABLE 5: The item weights and values in the $0-1$ knapsack problem.

a node $N$ into $N_{1}$ and $N_{2}$, we want one node to have the maximum number of characters on the splitting dimension in its DMBR, while the other node to have the minimum number of characters. And both new nodes must satisfy the minimum space utilization criterion in our example. If we solve the 01 knapsack problem as mentioned above, it will give us the best candidate partition (according to proposed heuristic R3) for splitting the node $N$ as shown in table 6 . Note that for a

\begin{tabular}{l|c}
\hline Entries in node $N_{1}$ & $G_{1}, G_{2}, G_{4}, G_{5}, G_{6}$ \\
\hline Entries in node $N_{2}$ & $G_{3}$ \\
\hline
\end{tabular}

TABLE 6: The candidate partition for an overflow node $N$ found by solving the 0-1 knapsack problem.

leaf node, the optimal solution to this splitting problem is even simpler since all the entries in the overflow leaf node have only a single character on a splitting dimension. This is a special case of the 0-1 knapsack problem which could be solved using a greedy algorithm (instead of dynamic programming) as follows. We first sort all items based on their weights. Then we put those sorted items into a knapsack $K$ (new tree node $N_{1}$ ) one by one, starting from the items with smaller weights until no more item could be put into $K$. All the remaining items are put into tree node $N_{2}$. This distribution approach will guarantee to obtain the best partition of entries in an overflow leaf node as required by $\mathrm{R} 3$.

By mapping the node splitting problem to the 0-1 knapsack problem, our proposed BoND-tree's splitting algorithm is guaranteed to find an overlap-free partition satisfying the minimum utilization criterion as long as there exists such a partition. Theoretically there may be cases when it is simply impossible to get any overlap-free split without affecting the space utilization. To safeguard the situation, the BoND-tree generates a candidate partition for each dimension by putting as many entries as possible to a new node based on the descending order of $r_{i}=v_{i} / w_{i}$, where $v_{i}$ is the cardinality of an entry $E_{i}$ 's $(1 \leq i \leq n, n$ is the total number of entries in the node) component set on the splitting dimension and $w_{i}$ is the storage space of $E_{i}$. Then we use heuristic R1 to pick one candidate partition which gives the least overlap value. In other words, only heuristic R1 is used when no overlap-free partition exists for an overflow node (a random one is chosen if there are ties for R1).

Note that, because of the nature of the NDDS as we described in section 4.4, in most splits the BoND-tree could find at least one overlap-free partition for an overflow node. Table 7 shows the percentage of non-overlap-free splits (i.e., no overlap-free partition could be found) among the total number of splits in our experiments with synthetic data. These experiments are described in detail in section 7. In our experiments with real data, it was observed that an overlap free partition was found in all the splits. This is due to the fact that real data has more dimensions (21 dimensional q-grams from genome sequences) and, therefore, has significantly more possibility of finding overlap-free partitions.

In algorithm 1 , if a solution is returned in line 16 , it is guaranteed to be an overlap-free partition which satisfies the minimum utilization criterion. Otherwise the code segment between lines $19 \sim 22$ finds (and returns) a partition which is not overlap-free but satisfies the minimum utilization criterion. 


\begin{tabular}{c|c}
\hline Number of vectors indexed & Percentage of non-overlap-free splits \\
\hline $1 \mathrm{M}$ & $0.552 \%$ \\
$2 \mathrm{M}$ & $0.618 \%$ \\
$3 \mathrm{M}$ & $0.577 \%$ \\
$4 \mathrm{M}$ & $0.586 \%$ \\
$5 \mathrm{M}$ & $0.558 \%$ \\
\hline
\end{tabular}

TABLE 7: The percentage of non-overlap-free splits when building the BoND-tree

\subsection{Deletion in the BoND-tree}

If removing a vector from a leaf node $L$ does not cause any underflow (i.e., the minimum space utilization requirement on $L$ is satisfied after the deletion), the vector is directly removed and DMBRs of $L$ 's ancestor nodes are adjusted as needed. If an underflow occurs for $L$, the procedure is described as follows.

Node $L$ is removed from its parent node $N$, and if $N$ underflows again, $N$ is removed from its parent node. The procedure propagates toward the root until no underflow occurs. Then the subtree represented by the underflow node closest to the root node is removed, its ancestor nodes' DMBRs are adjusted as needed and all the remaining vectors in the subtree are reinserted. In the worst case, if the root node has only two children and one of them is removed, the remaining child node becomes the new root of the tree (i.e., tree height decreases by one).

An update operation can be implemented as a combination of deletion and insertion. In order to update a vector, we first delete it from the database, and insert the modified vector.

\subsection{Box Query on the BoND-tree}

The algorithm for executing box queries on the BoND-tree is implemented as follows. Let $q$ be the query box and $N$ be a node in the tree (which is initialized to root $R$ of the tree). For each entry $E$ in $N$, if the query window $w$ overlaps with the DMBR of $E$, entry $E$ is searched. Otherwise, the subtree rooted at $E$ is pruned.

\section{Compression technique for the BOND-TREE}

We now present a possible improvement in the BoND-tree structure using node compression.

\subsection{Motivation}

In the CDS, the minimum bounding rectangle (MBR) information on a continuous dimension is stored by recording the lower and upper bounds of that dimension. Since the number of available values in a continuous domain is usually unlimited (or very large), the MBR information on a continuous dimension $i$ in a hierarchical indexing structure (e.g., the $\mathrm{R}^{*}$ tree) is unlikely to cover the whole domain of $i$. However, in the NDDS the number of characters in a discrete domain is limited (and typically quite small). This means a discrete dimension for a DMBR will get full (i.e., all characters in the domain have appeared on that dimension) much faster than a continuous dimension.

Consider a set $S$ which contains characters from a nonordered discrete domain $D$ with domain size $|D|=A$. The
Markov transition matrix [19] describing the probability of $S$ 's size after adding one random character from $D$ to $S$ is shown in (20).

$$
P=\begin{gathered}
1 \\
1 \\
2 \\
3 \\
\ldots \\
A
\end{gathered}\left(\begin{array}{ccccc}
1 / A & (1-1 / A) & 3 & \ldots & A \\
0 & 2 / A & (1-2 / A) & \ldots & 0 \\
0 & 0 & 3 / A & \ldots & 0 \\
\ldots & \ldots & \ldots & \ldots & \ldots \\
0 & 0 & 0 & \ldots & 1.0
\end{array}\right)
$$

Now suppose we are creating an indexing structure for an NDDS with domain $D$ for dimension $i$. Further suppose the size of $D$ is 10 . Using the Markov transition matrix in (20), we can calculate the probability of a node $N$ having all the 10 characters in $D$ on dimension $i$ after indexing $V_{n}$ vectors, as shown in table 8 .

\begin{tabular}{c|ccccc}
\hline$V_{n}$ & 20 & 40 & 60 & 80 & 100 \\
\hline Probability & $21.47 \%$ & $85.81 \%$ & $98.21 \%$ & $99.78 \%$ & $99.97 \%$ \\
\hline
\end{tabular}

TABLE 8: Probability of having a full dimension after indexing $X$ vectors.

As we can see from the table, after indexing 100 vectors, the probability that all the 10 characters in $D$ have appeared in node $N$ 's DMBR on dimension $i$ is $99.97 \%$. And it will become even higher for a smaller alphabet size (i.e., $|D|<10$ ) or a larger number of vectors $(X>100)$.

The splitting heuristics of the BoND-tree prefer an overlapfree candidate partition generated from a shorter dimension. This leads to more full dimensions in the DMBRs of non-leaf nodes of the BoND-tree (especially at higher levels of the tree) compared to the ND-tree. Table 9 shows the percentage of full dimensions in the non-leaf nodes' DMBRs when indexing 5 million vectors from 16 -dimensional NDDSs with varying alphabet sizes. From the above statistics, we see that a large percentage of dimensions recorded in the DMBRs of non-leaf nodes are full in the BoND-tree. This fact can be exploited to reduce the amount of space required to store the DMBR. In the following subsections we explain our compression scheme and its effect on the node splitting algorithm.

\subsection{The Compressed BoND-tree Structure}

In a non-leaf node entry of the compressed BoND-tree, we use one additional bit to indicate if the DMBR is full or not on each dimension. Only when it is not full, we record the occurrence of each character on that dimension. As the space requirement of a single DMBR is reduced, the fanout of the node increases. This high fanout results in reduction in the height of the tree and reduced I/O at the time of querying.

\begin{tabular}{c|cccc}
\hline Alphabet size & 10 & 15 & 20 & 25 \\
\hline$\%$ of full dimensions & $75.33 \%$ & $75.44 \%$ & $79.04 \%$ & $81.30 \%$ \\
\hline
\end{tabular}

TABLE 9: Percentage of full dimension at non-leaf levels of the BoND-tree with different alphabet sizes. 
Note that the compression of DMBRs applies only to non-leaf nodes because the leaf node entry in the BoNDtree has only one character along each dimension. Thus the performance gain of the compressed BoND-tree is achieved through a more effective representation of DMBRs in the nonleaf nodes, especially nodes at higher levels of the tree.

\subsection{Effect of Compression on Splitting Overflow Non-leaf Nodes}

When a non-leaf node entry's DMBR is split along one dimension, the resulting DMBRs may also shrink along other (full) dimensions. Thus those previously compressed (omitted) dimensions may become uncompressed, leading to more space required. This may give rise to a concern whether two new nodes are sufficient to hold all the entries from splitting an overflow node. However, it is not difficult to see that this is not a problem.

In a non-leaf node $N$, the need for its splitting comes when one of its node entries $E$ gets replaced with two new entries $E^{\prime}$ and $E^{\prime \prime}$ (due to the split of a child node $N_{E}$ ).

The entries in $N$ that need to be stored after splitting $N_{E}$ are: $E^{\prime}, E^{\prime \prime}$, and all original entries in $N$ except $E$. If $N$ does not have enough space for these entries, it needs to be split. In the worse case (i.e., no dimension in DMBRs of $E^{\prime}$ and $E^{\prime \prime}$ could remain compressed), the space required for storing all the entries from splitting $N$ is equal to the space needed for storing all original entries in $N$ except $E$ plus the space required to hold two uncompressed entries $\left(E^{\prime}\right.$ and $\left.E^{\prime \prime}\right)$. As any node must be able to hold at least two uncompressed node entries for indexing to be possible, two new nodes are sufficient for holding all the entries in the overflow node.

\section{EXPERIMENTAL RESULTS}

To evaluate the performance of the BoND-tree we conducted extensive experiments. The results are reported in this section.

\subsection{Experimental setup}

The BoND-tree was implemented in C++. Experiments were conducted on machines with Intel Xeon quad-core processors with 8 GB ECC DDR2 RAM running SuSE Enterprise Linux 10 in a high performance computing cluster system.

Performance of the proposed BoND-tree (with and without compression) was evaluated using synthetic data with various dimensions, alphabet sizes and database sizes (the number of vectors indexed). We generated uniform and skewed (Zipfian) data for the experiments. Each data record is generated by randomly generating a letter in each dimension. The probability of each letter in the alphabet is the same for uniform data (so for alphabet size of 10, each letter will have probability of 0.1). For Zipfian data, probability of each letter is inversely proportional to its rank among all the letters in the alphabet. For example, let $\{a, b, c\}$ be the alphabet for a certain dimension and let ranks of letters $a, b, c$ be $1,2,3$ respectively. Then the probability of these three letters will be $0.55,0.27$ and 0.18 respectively. Besides the evaluation based on synthetic data sets, we also used real data for performance comparison of box queries. In each of the tests, 200 random box queries were executed and the average number of $\mathrm{I} / \mathrm{O}$ and average running time was measured. As box queries are the focus of this paper, we do not present results on range (similarity) queries. However we would like to note that the ND-Tree provides better performance than the BoND-tree for range queries.

To the best of our knowledge so far there has been no indexing technique specifically designed to support efficient box queries in the NDDS. Query performance of the BoND-tree was compared with that of the ND-tree, Data decomposition (DD), the $10 \%$ linear scan and the M-tree.

The ND-tree is an indexing scheme designed exclusively for range queries in the NDDS, which is reported to be a robust technique compared to other known indexing methods in NDDS [20]. Since the sequential scan (i.e., flat files without indexing) is much faster than the random disk access needed for indexing, $10 \%$ of the total $\mathrm{I} / \mathrm{O}$ needed for sequential scan [6], [20], [26] is used to compare with that of the BoND-tree. The vertical data decomposition scheme discussed in [10] has an effective strategy for the nearest neighbor search. However, for a box query it may be very difficult to come up with a good pruning strategy. Hence, even though this method is conceptually similar to BoND-tree heuristics, it fails to provide any improvement in the search performance. Our experiments show that this strategy is worse than the $10 \%$ linear scan in most of the cases. The M-tree was designed for the metric spaces. Although it could be utilized to support indexing of the NDDS, its performance is quite poor. Our experimental results show that the M-tree needs more I/O than the $10 \%$ linear scan to support box queries in the NDDS. Since M-tree and DD are not optimized for the NDDS and are found to be worse than linear scan, we do not consider their performance in rest of performance comparisons.

\subsection{Tree construction time}

Figure 1 compares construction time of BoND-tree with that of ND-tree for increasing database sizes. It can be seen that building BoND-tree takes much more time than building the ND-tree. This is not surprising since BoND-tree insertion algorithm is fairly complex compared to that of ND-tree.

\subsection{Impact of each heuristic on performance}

Figure 2 compares the query I/O when heuristic R1 alone, $\mathrm{R} 1$ followed by $\mathrm{R} 2$ and $\mathrm{R} 1$ followed by $\mathrm{R} 2$ followed by $\mathrm{R} 3$

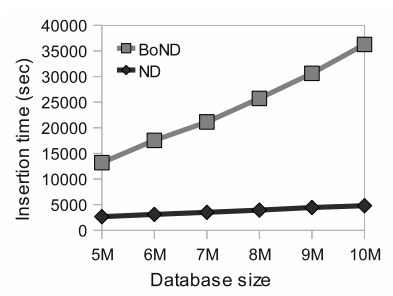

Fig. 1: Comparison of construction times of BoND-tree and ND-tree 


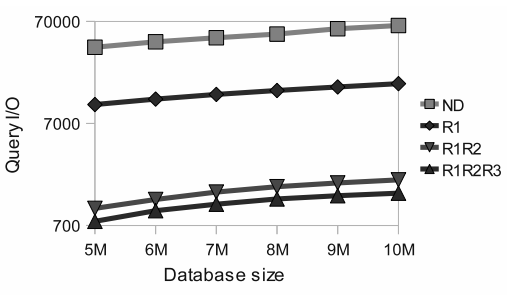

Fig. 2: Improvement due to each of the heuristics in query I/O

are used. We also include I/O for ND-tree (labeled 'ND') as the baseline for comparison. It can be seen that each heuristic helps in reducing the I/O for the query. Heuristic R1 alone provides about $75 \%$ improvement in I/O over ND-tree. Combination of R1 and R2 provides further improvement of about $80 \%$ over R1. The combination R1-R2-R3 reduces I/O even further by about $30 \%$ over R1-R2. This clearly justifies the use of all three heuristics.

\subsection{Effect of Different Database Sizes}

In this set of tests we evaluate the performance of the BoNDtree for different database sizes. We varied the number of indexed data vectors from 5 millions to 10 millions. The data set used for the tests has 16 dimensions and the alphabet size for each dimension is 10 . The average query I/O performance for box size 2 is shown in figure $3 a$. It can be seen that, as the number of indexed data points increases, the query I/O increases for all the techniques in our tests. However, the BoND-tree is a clear winner for all database sizes. The average query $\mathrm{I} / \mathrm{O}$ for the BoND-tree is several orders of magnitude smaller than that of the ND-tree. The total time for BoNDtree was much better than that for ND-tree. However, due to space constraints, we could not include any tables/graphs in the paper.

\subsection{Effect of Different Numbers of Dimensions}

This set of tests evaluates the performance of the BoND-tree when indexing data sets with different numbers of dimensions (see figure $3 \mathrm{~b}$ ). In the experiments, the number of dimensions was varied from 8 to 20 . Other parameters such as the database size, the alphabet size and the query box size were kept constant at 5 millions, 10 and 2, respectively. With the increasing number of dimensions, more space is required to store the DMBR information in the BoND-tree as well as in the ND-tree. This results in reduction of the fanout of tree nodes and a subsequent increase in the height of the tree. Thus, the $\mathrm{I} / \mathrm{O}$ for both trees (as well as the 10\% linear scan) increases. The relative number of I/O for the BoND-tree is much less than both the ND-tree and the $10 \%$ linear scan. Further, as figure $3 \mathrm{~b}$ shows, the BoND-tree is much less affected by the increased number of dimensions than the ND-tree.

\subsection{Effect of Alphabet Size}

In this set of tests, the alphabet size was varied from 10 to 30 in steps of 5. Figure $3 \mathrm{c}$ shows performances of the BoND-tree, the ND-tree and the $10 \%$ linear scan for various alphabet sizes.
As the alphabet size increases, the ability of the tree to find an overlap-free partition increases which results in a decrease in the I/O. The number of dimensions of indexed vectors was 16 . The database size and query box size were 5 million and 2 , respectively.

\subsection{Effect of Different Query Box Sizes}

This set of tests compares the performance of the BoND-tree with those of the ND-tree and the $10 \%$ linear scan for different box sizes. The number of dimensions and the alphabet size were fixed at 16 and 10 , respectively. We experimented with both uniform boxes (i.e., all the sides have the same length) as well as non-uniform boxes (sides of the box are chosen randomly).

\subsubsection{Uniform Boxes}

For this set of experiments, the database size was fixed at 5 millions and the box size was increased from 1 to 5 . As the query box size increases, both the BoND-tree and the NDtree require more $\mathrm{I} / \mathrm{O}$ while the number of $\mathrm{I} / \mathrm{O}$ for the $10 \%$ linear remains constant. As we can see from figure $3 d$, the performance gain of the BoND-tree is significant for all box sizes given. Our proposed BoND-tree maintains its superior performance even at a box size of 5 . For larger box sizes however, the $10 \%$ linear scan proves to be the best method. This is expected as the result set is huge when the query box size is large, in which case, no index is beneficial.

\subsubsection{Non-uniform Boxes}

This section compares the performance of the BoND-tree for non-uniform box sizes. We varied the database size from 5 million to 10 million records. A query box is generated by randomly selecting an edge length along each dimension. The maximum edge length was limited to 5 (i.e., $50 \%$ of alphabet size). We generated 200 such queries and calculated the average query I/O. Figure 3e shows our findings. It can be seen that BoND-tree significantly outperforms both the other schemes.

\subsection{BoND-tree with skewed data}

Figure $3 \mathrm{f}$ shows the effect of applying BoND-tree and ND-tree to skewed data (having Zipf distribution) for increasing box size. The database size was set to 5 million and the number of dimensions was 16 . It should be noted that the BoND-tree is significantly better than the ND-tree or the linear scan even for relatively large box size of 5 . This demonstrates effectiveness of BoND-tree in non-uniform data spaces.

\subsection{Application in Primer Design}

As explained earlier, box queries in NDDS are useful in primer design for genome sequence databases. In this section we present results of applying the BoND-tree for this application.

In order to enable a sub-sequence search, the index is built of all possible overlapping sub-sequences (Q-grams) of a genome sequence having the given primer length. Hence, the actual data needed to create the index is several times more 


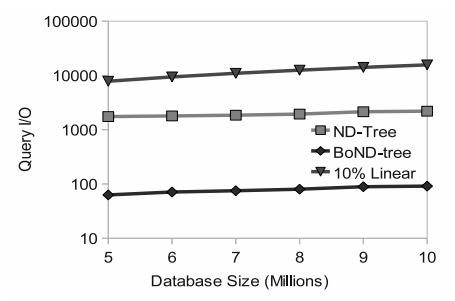

(a) Database size

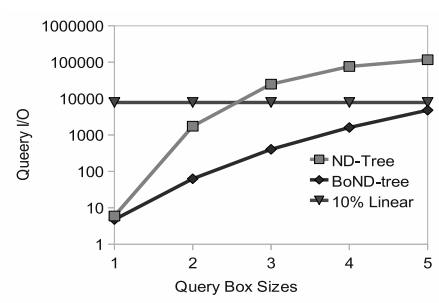

(d) Uniform query box size

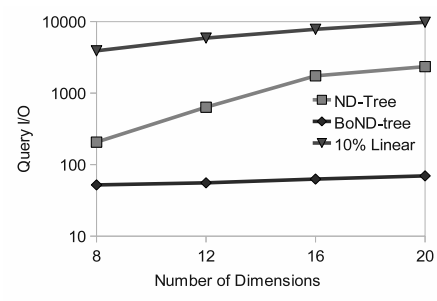

(b) Number of dimensions

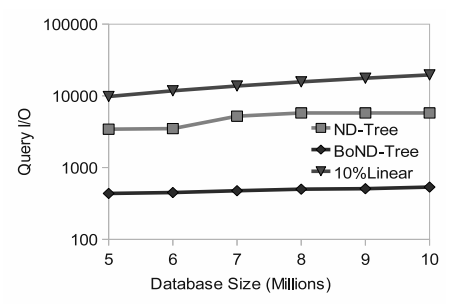

(e) Non-uniform query boxes

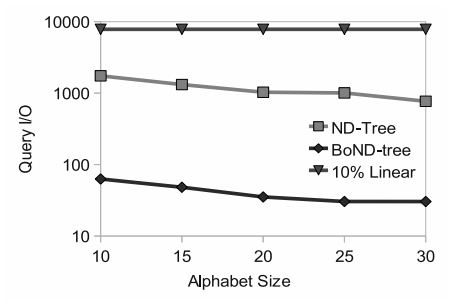

(c) Alphabet size

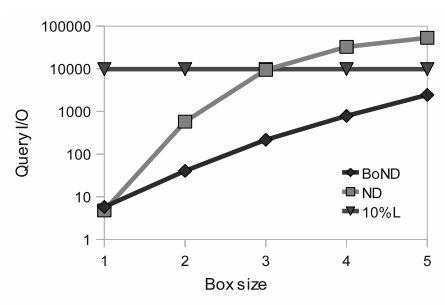

(f) Skewed (zipf) data

Fig. 3: Experimental evaluation of impact of various parameters on performance of BoND-tree

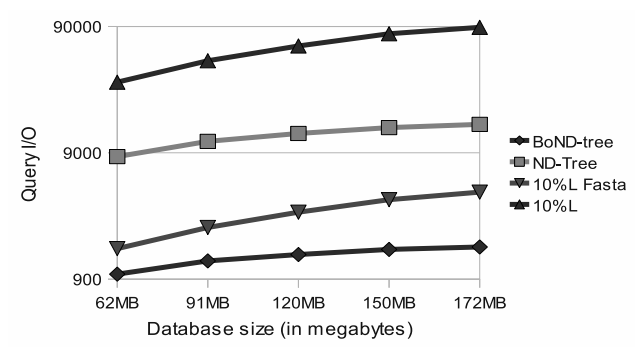

(a) Query I/O

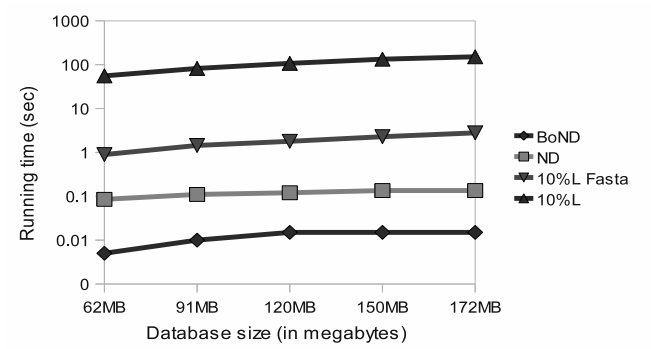

(b) Running time

Fig. 4: Performance of indexing genome sequence data

than the sequence data. But despite the increased index size, searching is remarkably efficient in the BoND-tree.

We carried out experiments with varying sizes of genome sequence databases. The smallest database contains 50 thousand genome sequences while the largest one contains 150 thousand sequences. The database size was increased in steps of 25 thousand sequences. Simple fasta file (which is the standard file format used in computational biology) was used as the input. Figure 4 shows the number of I/O and query running time for each of the schemes. The BoND-tree and the ND-Tree were built for overlapping Q-grams. We calculated I/O for $10 \%$ linear scan using fasta file as the input (labeled '10\% Linear Fasta' in the figure) as well as Q-grams as input (labeled ' $10 \%$ Linear'). It can be seen that the BoND-tree is by far the best indexing scheme. In fact, as the number of indexed sequences increases, improvement due to the BoNDtree also increases. For the largest database ( 1 ize $=172 \mathrm{MB})$ containing 150000 sequences, BoND-tree provides about $60 \%$ improvement. This highlights importance of BoND-tree in certain class of applications.

\subsection{Comparison of running time}

Experiments so far show that in terms of number of disk page accesses (or query I/O), BoND-tree significantly outperforms ND-tree as well as linear scan under various conditions. Since query $\mathrm{I} / \mathrm{O}$ is the major contributor in running time for any index based query, we expect BoND-tree to perform much better in terms of query execution time as well. In this section, we present the results confirming superior running time of queries in BoND-tree. The hardware and setup used for these experiments are already described in section 7.1. Unless explicitly specified otherwise, database size, box size and the number of dimensions were set to 5 million, 4 and 16 respectively. As shown in the figures 5a through 5f, query execution time of BoND-tree is considerably smaller than that of linear scan or ND-tree.

\subsection{Performance of the Compressed BoND-tree}

We also examined the performance of BoND-tree using the proposed compression strategy. First we show the performance gain for varying number of dimensions. The database size used for this set of tests is 5 millions. The query box size and the alphabet size are set to 2 and 10, respectively. As we can see from figure $6 \mathrm{a}$, for all the test cases, the BoNDtree without compression of DMBR uses more than $10 \%$ of I/O than the compressed BoND-tree to answer the same queries. Figure $6 \mathrm{~b}$ shows the performance of the compressed BoND-tree for different alphabet sizes. The number of vectors indexed is fixed at 5 millions, the number of dimensions is set to 16 and the query box size is 2 . This set of tests demonstrates the effectiveness of the compression strategy when indexing NDDSs with different alphabet sizes. Although 


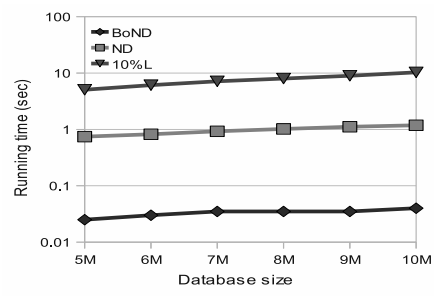

(a) Database size

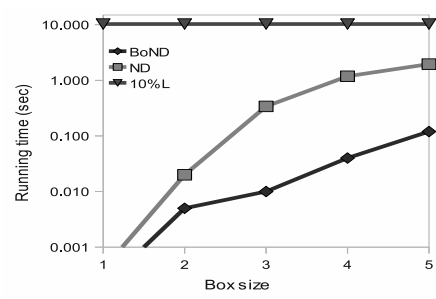

(d) Uniform query box size

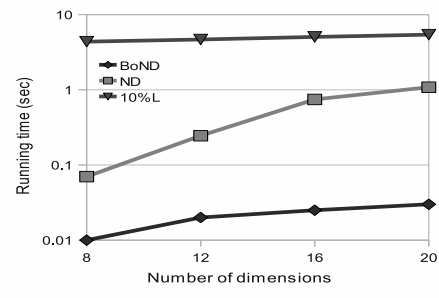

(b) Number of dimensions

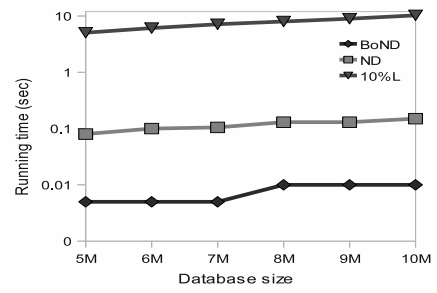

(e) Non-uniform query boxes

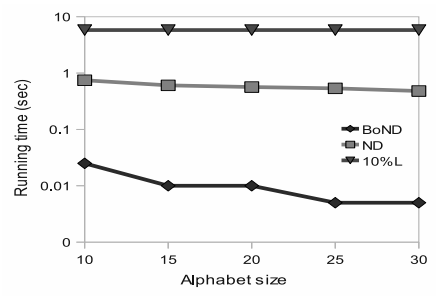

(c) Alphabet size

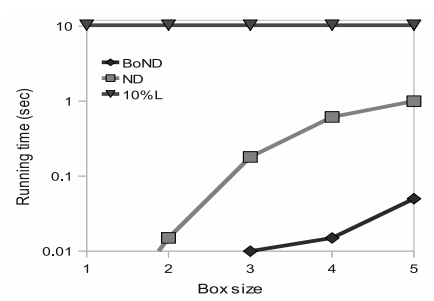

(f) Skewed(zipf) data

Fig. 5: Comparison of running time of the queries for various parameters

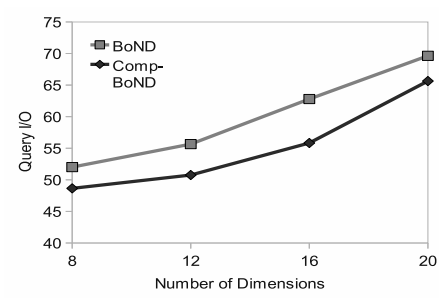

(a) Number of dimensions

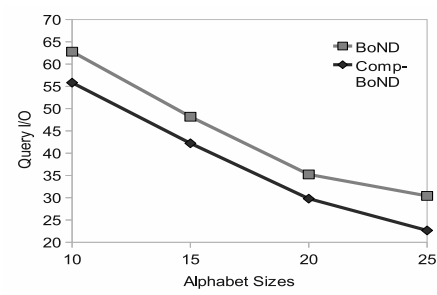

(b) Alphabet size

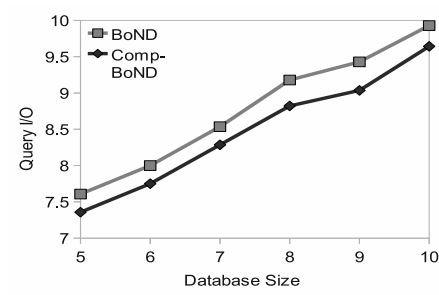

(c) Non-uniform queries

Fig. 6: Performance comparison of the Compressed BoND-tree with uncompressed BoND tree

\begin{tabular}{c|c|c}
\hline Box size & BoND-tree & Compressed BoND-tree \\
\hline 2 & 39.8571 & 36.8929 \\
3 & 226.857 & 219.286 \\
4 & 822.571 & 803.429 \\
5 & 2210.57 & 2171.68 \\
\hline
\end{tabular}

TABLE 10: Performance of the Compressed BoND-tree for uniform box queries.

both compressed and uncompressed indexing methods yield lesser $\mathrm{I} / \mathrm{O}$ as the alphabet size grows, the compressed one outperforms the uncompressed one for all the alphabet sizes used in the experiments. Table 10 and figure $6 \mathrm{c}$ show the comparison of compressed BoND-tree for uniform and nonuniform box queries, respectively. In table 10, the database size and the number of dimensions were kept constant at 5 million and 16, respectively. As can be seen from the table, the compressed BoND-tree is consistently better than the basic BoND-tree. However, as the box size increases the amount of data space being queried increases exponentially, which results in both the trees approaching performance of linear scan. In figure $6 \mathrm{c}$, the database size was increased from 5 million to 10 million records. As expected, the compressed BoND-tree consistently performs better than the uncompressed BoNDtree. These results highlight the advantages of the proposed compression technique.

\section{Conclusion}

In this paper, we have presented a new indexing structure, called the BoND-tree, which exploits exclusive properties of the NDDS. Theoretical analysis of box queries in the NDDS shows that a better filtering power could be achieved using new splitting heuristics adopted by the BoND-tree. Our extensive experimental results using different alphabet sizes, database sizes, dimensions and query box sizes demonstrate that the BoND-tree isscp bon significantly more efficient than existing techniques such as the ND-tree and the $10 \%$ linear scan. Effectiveness of the BoND-tree in a real world application involving genome sequence databases is demonstrated. We also present the use of compression in the NDDS to further improve performance of the BoND-tree.

\section{ACKNOWLEDGMENT}

Research supported by the US National Science Foundation (under grants \#IIS-0414576 and \#IIS-0414594), the Michigan State University and the University of Michigan. We wish to acknowledge the support of the Michigan State University High Performance Computing Center and the Institute for Cyber Enabled Research. The authors would like to thank Dr. James Cole and Dr. Benli Chai and Mr. Jordan Fish, who work 
for Ribosomal Database Project (RDP) under Grant No. DEFG02-99ER62848 supported by the Office of Science of U.S. Department of Energy, for their valuable suggestions and help. The authors also acknowledge Dr. Gang Qian for his help.

\section{REFERENCES}

[1] R. Bayer and K. Unterauer, "Prefix B-trees," ACM Transactions on Database Systems, pp. 11-26, 1977.

[2] N. Beckmann, H.-P. Kriegel, R. Schneider, and B. Seeger, "The R*tree: an efficient and robust access method for points and rectangles," Proceedings of ACM SIGMOD, pp. 322-331, 1990.

[3] J. L. Bentley and R. Sedgewick, "Fast algorithms for sorting and searching strings," Proceedings of the eighth annual ACM-SIAM symposium on Discrete algorithms, pp. 360-369, 1997.

[4] S. Berchtold, D. Keim, and H.-P. Kriegel, "The X-tree: an index structure for high-dimensional data," Proceedings of the 22nd International Conference on VLDB, pp. 28-39, 1996.

[5] T. Bozkaya and M. Ozsoyoglu, "Indexing large metric spaces for similarity search queries," ACM Transactions on Database Systems, vol. 24, no. 3, pp. 361-404, 1999.

[6] K. Chakrabarti and S. Mehrotra, "The hybrid tree: an index structure for high dimensional feature spaces," Proceedings of the 15th International Conference on Data Engineering, pp. 440-447, 1999.

[7] E. Chávez, G. Navarro, R. Baeza-Yates, and J. L. Marroquín, "Searching in metric spaces," ACM Comput. Surv., vol. 33, no. 3, pp. 273-321, 2001.

[8] P. Ciaccia, M. Patella, and P. Zezula, "M-tree: An efficient access method for similarity search in metric spaces," pp. 426-435, 1997.

[9] J. Clément, P. Flajolet, J. Clément, B. Vallée, B. Vallée, T. G. Logiciel, and P. Algo, "Dynamical sources in information theory: A general analysis of trie structures," Algorithmica, vol. 29, pp. 307-369, 1999.

[10] A. P. de Vries, N. Mamoulis, N. Nes, and M. Kersten, "Efficient k-nn search on vertically decomposed data," pp. 322-333, 2002.

[11] M. E. D. El Baz, "Load balancing in a parallel dynamic programming multi-method applied to the 0-1 knapsack problem," pp. 127-132, 2006.

[12] P. Ferragina and R. Grossi, "The string B-tree: a new data structure for string search in external memory and its applications," Journal of the $A C M$, pp. 236-280, 1998.

[13] A. Guttman, "R-Trees: a dynamic index structure for spatial searching," Proceedings of ACM SIGMOD, pp. 47-57, 1984.

[14] A. Henrich, "The LSDh-tree: an access structure for feature vectors," Proceedings of the 14th International Conference on Data Engineering, pp. 362-369, 1998.

[15] G. R. Hjaltason and H. Samet, "Index-driven similarity search in metric spaces (survey article)," ACM Trans. Database Syst., vol. 28, no. 4, pp. 517-580, 2003.

[16] D. E. Knuth, The Art of Computer Programming, Volume III: Sorting and Searching. Addison-Wesley, 1973.

[17] A. Liu, J. Wang, G. Han, S. Wang, and J. Wen, "Improved simulated annealing algorithm solving for 0/1 knapsack problem," pp. 1159-1164, 2006.

[18] W. Loots and T. H. C. Smith, "A parallel algorithm for the 0-1 knapsack problem," Int. J. Parallel Program., vol. 21, no. 5, pp. 349-362, 1992.

[19] S. Meyn and R. Tweedie, Markov Chains and Stochastic Stability. Springer-Verlag, 1993.

[20] G. Qian, Q. Zhu, Q. Xue, and S. Pramanik, "The ND-tree: a dynamic indexing technique for multidimensional non-ordered discrete data spaces," pp. 620-631, 2003.

[21] G. Qian, Q. Zhu, Q. Xue, and S. Pramanik, "Dynamic indexing for multidimensional non-ordered discrete data spaces using a data-partitioning approach," ACM Trans. Database Syst., vol. 31, pp. 439-484, June 2006.

[22] J. Robinson, "The K-D-B-tree: a search structure for large multidimensional dynamic indexes," Proceedings of ACM SIGMOD, pp. $10-18$, 1981.
[23] T. J. Rolfe, "An alternative dynamic programming solution for the $0 / 1$ knapsack," SIGCSE Bull., vol. 39, no. 4, pp. 54-56, 2007.

[24] S. Sahni, "Approximate algorithms for the 0/1 knapsack problem," $J$. $A C M$, vol. 22, no. 1, pp. 115-124, 1975.

[25] G. Salton, A. Wong, and C. S. Yang, "A vector space model for automatic indexing," Communications of the ACM, vol. 18, no. 11, pp. 613-620, November 1975.

[26] R. Weber, H. J. Schek, and S. Blott, "A quantitative analysis and performance study for similarity-search methods in high-dimensional spaces," pp. 194-205, 1998.

[27] P. Weiner, "Linear pattern matching algorithms," Proceedings of the 14th Annual Symposium on Switching and Automata Theory, pp. 1-11, 1973.

[28] S. K. Wong, W. Ziarko, V. V. Raghavan, and P. C. Wong, "On modeling of information retrieval concepts in vector spaces," ACM Transactions on Database Systems, vol. 12, no. 2, pp. 299-321, 1987.

[29] P. N. Yianilos, "Data structures and algorithms for nearest neighbor search in general metric spaces," pp. 311-321, 1993.

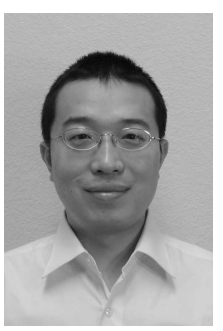

Changqing Chen is currently a senior Engineer working at Yahoo! Inc. He received his PhD degree from the Computer Science and Engineering Department at Michigan State University, and Bachelor's degree from Peking University. His research interests include large scale data processing and high-dimensional data indexing.

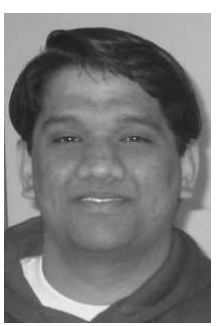

Alok Watve is a Ph.D. candidate in the Computer Science and Engineering Department at Michigan State University. He has a Master of Technlogy degree from Indian Institute of Technology Kharagpur. His research interests include database indexing, data mining and image processing.

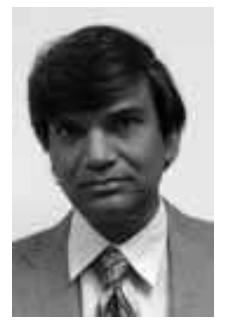

Sakti Pramanik received the BE degree in Electrical Engineering from Calcutta University and awarded University gold medal for securing the highest grade among all branches of Engineering. He received the MS degree from the University of Alberta, Edmonton, in electrical engineering, and the PhD degree in computer science from Yale University. He is currently a professor in the Department of Computer Science and Engineering at Michigan State University.

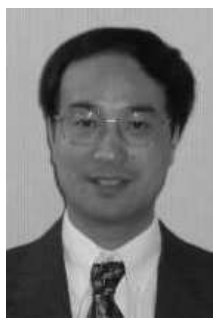

Qiang Zhu received his Ph.D. in Computer Science from the University of Waterloo (Canada) in 1995. He is currently a Professor in the Department of Computer and Information Science at The University of Michigan - Dearborn. $\mathrm{He}$ is also an IBM CAS Faculty Fellow at the IBM Toronto Lab and an IEEE Senior Member. His current research interests include query optimization, streaming data processing, multidimensional indexing, self-managing databases and Web information systems. 